BPMJ

4,1

10

\title{
Concurrent design for mass customization
}

\author{
Mitchell M. Tseng and Jianxin Jiao
}

Department of Industrial Engineering \& Engineering M anagement, The Hong Kong U niversity of Science \& Technology, Clear Water Bay, Kowloon, Hong Kong

\section{Introduction}

Concurrent engineering (CE) has been considered as one of the most effective approaches to reducing product development lead time and achieving overall cost savings (Sohlenius, 1992). How ever, recent trends of increasing product variety, primarily due to the growing areas of self expression and the explosive expansion of global markets, pose challenges to the traditional CE agenda (M olina et al., 1995). M anufacturers often have to struggle with the trade-off between time to market, product variety, and mass efficiency. The symptom manifests an emerging manufacturing paradigm, namely mass customization, which aims at satisfying individual customer needs with mass production efficiency (Pine, 1993).

M ass customization embarks a new paradigm for manufacturing industries (Pine, 1993). It recognizes each customer as an individual and provides each of them with attractive "tailor-made" features that can only be offered in the preindustrial craft system. In the meantime, its customers can afford the products because modern mass production enables low product cost. Thus with mass customization, companies can outpace their competitors in gaining new customers and achieving higher margins. Figure 1 illustrates the economic implications of mass customization. In high volume production, the volume is sufficient to defray the cost of investment in equipment, tooling, engineering, training, and others. M ass production clearly shows an advantage. How ever, in low to medium volume production, where production quantity cannot justify and leverage the investment, customers are otherwise willing to pay more because their special needs are satisfied. This is the area where we believe mass customization provides a tremendous advantage in business competition (T seng and Jiao, 1996).

Mass customization raises many implications for theory development and testing across a broad horizon. It is an attempt to summarize many recent trends towards manufacturing flexibility (greater variety and responsiveness to customers). A lthough the notion of pursuing a customization strategy has great 
\$/Unit

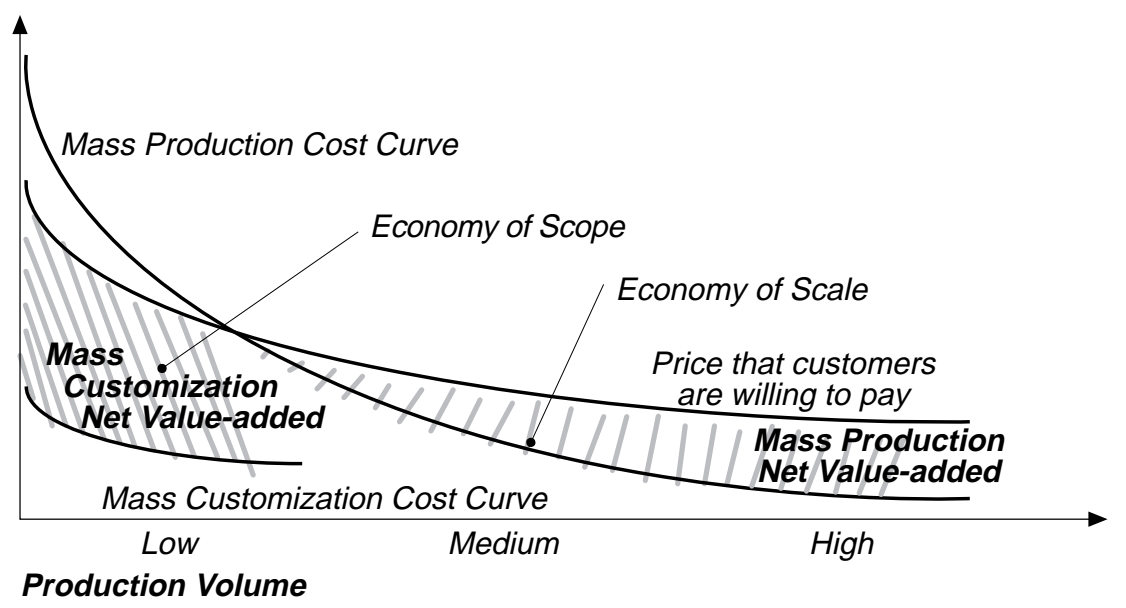

Concurrent design for mass customization 11

appeal, the current state-of-the-art literature on mass customization seems only to highlight the benefits rather than the means (K otha, 1995). A s a result, mass customization has drawn the criticism that it has the look and feel of a revolutionary paradigm, but that, without a coherent framework, it remains a repackaging of many ideas found in the manufacturing literature, with only limited synthesis (K otha, 1994). M oreover, competitive strategies in the 1990s include diverse and related themes such as manufacturing flexibility, timebased competition, lean production, re-engineering, and continuous improvement. The structure and infrastructure issues are critical to the successful implementation of mass customization with respect to the compatibility and balance with various strategies and technologies heretofore (Lau, 1995). A s a result, it is imperative to develop a coherent framework with systematic approaches to enable the realization of mass customization.

This paper tackles how to implement mass customization with the support of CE. The next section develops several critical requirements and technical challenges of mass customization. By discussing CE in the context of mass customization, the paper analyzes the potential means provided by CE for realizing mass customization in section 3 . Section 4 presents an approach to concurrent design for mass customization (CDFMC). The core of CDFMC is to develop a mass customization oriented product family architecture (PFA ) which works as a unifying, integrated platform for product creation and delivery. Section 5 presents a framework of PFA development. Following a case study given in section 6 , conclusions are drawn in section 7 .

\section{Technical challenges of mass customization}

The essence of mass customization lies in maximizing the congruence of the manufacturer's capabilities with the window of customer needs related to target market niches and in a timely manner, that is, a manufacturer has to 
BPM J

4,1

12

perceive and capture latent market niches and correspondingly develop its technical capabilities to meet diverse customer needs. Perceiving latent customization requires the exploration of market niches. The capture of target customer groups means emulating competitors in either quality or cost or quick response. To keep manufacturing costs low necessitates the appropriate development of production capabilities. Therefore, the requirements of mass customization lie in three aspects: time to market (quick responsiveness), variety (customization), and economies of scale (mass efficiency). The oxymoron of mass customization depends on a leverage of these requirements.

Resulting from these requirements, the main technical challenge in developing a coherent framew ork for mass customization lies in the ability to simultaneously satisfy the following requirements within a single approach (T seng and Jiao, 1996).

- Reusability/commonality. Optimizing reusability/commonality to achieve low cost and high efficiency, i.e. the economy of scale, an advantage characterized by mass production.

- Product platform. Providing a technical foundation for realizing customization, managing varieties, and leveraging core capabilities to optimize flexibility and foster a customer-focused and product-driven business.

- Integrated product development. Facilitating meta-level integration throughout the product development process and over product life-cycle to achieve quality and quick responsiveness.

\section{Concurrent engineering}

The essence of CE is concurrency and integration (Sohlenius, 1992). The achievement of time to market through telescoping lead times depends on the integration of design and production. To telescope the development time means carrying out development activities in parallel. Thus product design and process design engineering must be done simultaneously and symbolically, i.e. a continual to and fro between design and manufacture. The synergy between design and manufacturing produces a better and simpler product which is easier to manufacture and thus cheaper to produce, and in the meantime maximizes customer value, thus meeting the competitive challenge.

From the above explanations, it is not difficult to see that CE can provide remedies to some of the challenges in mass customization implementation. The key advantage is the integration of the product development processes and product life-cycle elements, thus obtaining lead time competitiveness and low product development cost. How ever, in terms of approaches and systems, current CE practice can only provide partial support to mass customization instead of the whole solution. In the context of mass customization, CE needs to further incorporate the following considerations. 
- Expanding organizational boundaries. Traditionally, most CE practice only focuses on the integration of design and manufacturing (Molina et al., 1995). M ass customization propounds the ever-increasing importance of sales and marketing. For example, the customers and suppliers may get involved as co-designers in sorting out the capability to satisfy their specific needs. Therefore, integrated design is required to cover the whole spectrum of the product development process. That is, the traditional integration boundary of CE has to be extended to encompass a larger scope spanning customer needs acquisition to product delivery.

- Optimizing reusability. The existing CE approaches and systems generally have no explicit answers to the economy of scale, rather emphasizing the reduction of lead time and development cost by integration and concurrency. How to identify commonality and building blocks to conduct design for reusability so as to maximize "common denominators" in design and production becomes an important dimension for CE to support mass customization.

- Integrating stand-alone design for " $X$ " tools. Even though $C E$ has been a research issue since the 1980s, most work has focused on the development of individual design for (DF) " $X$ " tools and systems (Molina et al., 1995). Design for " $X$ " embodies the concept of concurrent engineering, in which " $X$ " represents a series of engineering functions such as manufacturing, assembly, testability, and cost prediction. Towards the success of concurrent engineering, various design for " $X$ " tools must be integrated within a coherent framework (M olina et al., 1995).

- A chieving context-coherent integration. In recent years, research interest in feature-based modeling and reasoning has grown significantly to integ rate design and manufacturing. For a particular product, different specialists view it from different application (or engineering) perspectives and use different sets of features or feature decomposition. Therefore, a crucial problem in CE is the support of multiple feature contexts and the reasoning about features from one specific application perspective to another (Subrahmanian, 1991). Instead of conversion between feature-based representations, it is promising to support multiple viewpoints by developing unifying product and product family models to serve as the meta-integration platform and thus achieving the contextual coherence for CE integration.

\section{Concurrent design for mass customization}

In order to develop a framew ork by which a systematic approach can be taken to realize mass customization, we advocate concurrent design for mass customization (CDFM C) based on the belief that mass customization can be effectively approached from design, in particular the front-end design (T seng and Jiao, 1996). CDFM C aims at considering economies of scope and scale at the 
BPMJ

4,1

14

early design stage of the product realization process. The main emphasis of CDFMC is to elevate the current practice of designing individual products to designing product families. To support customized product differentiation, a product family platform is necessary to characterize customer needs and subsequently to fulfill these needs by configuring and modifying wellestablished modules and components. Therefore, there are two basic concepts undergirding CDFMC, namely family-based design (FBD) and product family architecture (PFA ). Figure 2 summarizes the conceptual implications of CDFM C in terms of the expansion of the context from both a design scope perspective and a product differentiation perspective.

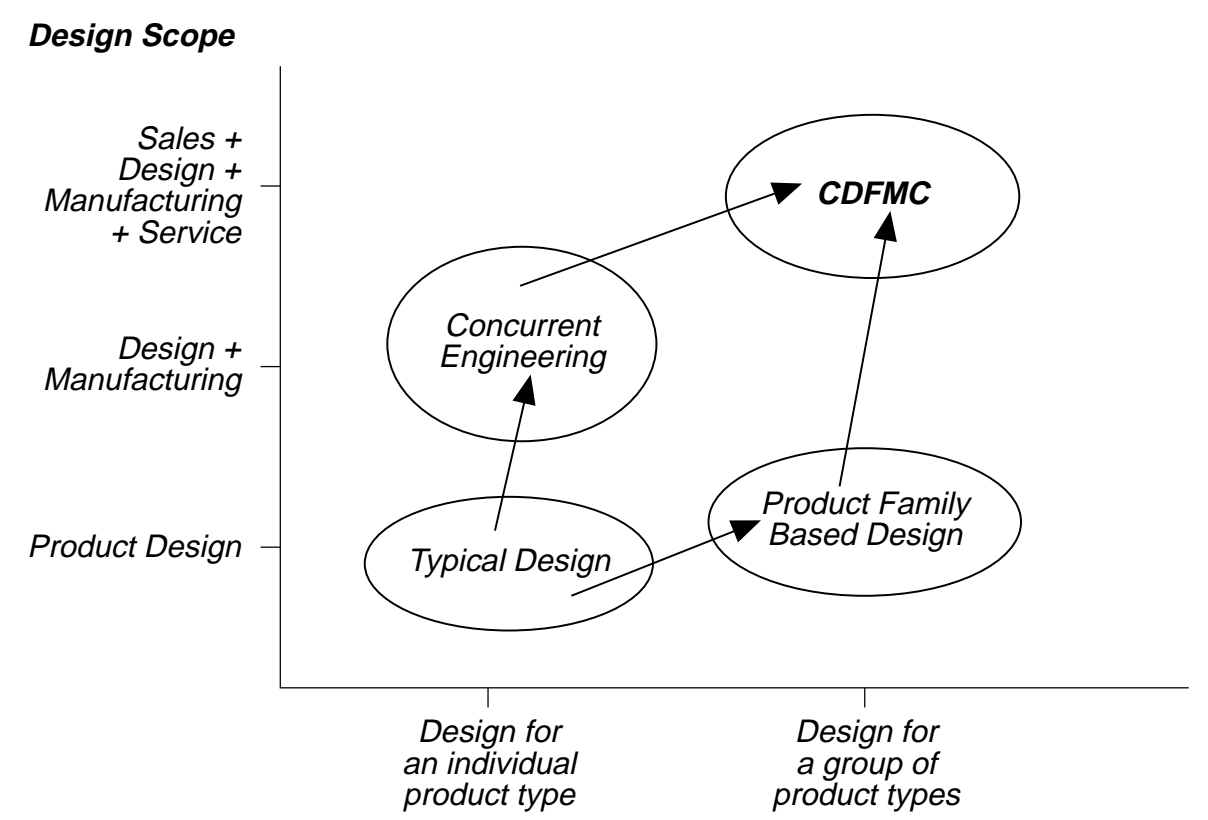

Product Differentiation

4.1 Family-based design

A product family is defined as a set of products that share common technology and address a related set of market applications (M eyer et al., 1993). T he commonality of technologies and markets leads to efficiency and effectiveness in manufacturing, distribution, and service, where the firm tailors each general resource or capability to the needs of specific market niches.

The essence of designing product families for mass customization is to identify and exploit the commonality among design solutions and manufacturing processes. The commonality captured is then built into a product platform to support the generation of new product variants so as to foster FBD. The merits of F BD lie in the increase of design rationale and 
manufacturing productivity, and the reduction of design and production costs. Through optimizing reusability, for perceived market niches, design changes and production set-ups are kept at a minimum level accompanied by maintaining a necessary variety portfolio. As a result, the advantages of mass production efficiency and low costs can be obtained.

4.2 Product family architecture

Concurrent design for mass customization

It has been shown that individual products can be efficiently constructed on successful generations of underlying product architectures, commonly referred to as the product platform. The technological foundation of product families is defined as the product platform (M eyer et al., 1993). A platform is the physical implementation of a technical design that serves as the base architecture for a series of derivative products. The platform also embraces the manufacturing technologies and processes employed in production. A strong platform provides leverage in the sense that each new derivative product can be developed at an incremental cost relative to the development of the initial product architecture.

In essence, a PFA means the underlying architecture of a product platform, within which various product variants can be derived from the basic product designs to satisfy a spectrum of customer needs related to various market niches. In other words, a good PFA provides a generic architecture to capture and utilize commonality, within which each new product instantiates and extends so as to anchor future designs to a common product line structure. In the context of mass customization, the rationale of a PFA lies in not only unburdening the knowledge base from keeping variant forms of the same solution, but also modeling the design process of a class of products that can widely variegate designs based on individual customization requirements within a coherent framework.

As the underpinning platform for FBD, a PFA is confronted with several structural issues, resulting from the variety of customized requirements, design solutions, and product structures. Figure 3 shows the multiple views of a PFA in terms of its structural elements, viz., product line structure, building blocks, and synthesis mechanism.

\section{Development of product family architecture}

The basis of PFA development derives from the understanding of the design process stated by axiomatic design theory (Suh, 1990). It defines a design world consisting of four distinctive domains, viz., the customer, functional, physical, and process domains. It also points out that design involves a zig-zagging mapping process between domains with creative conceptualization. Four domains are characterized by customer needs, functional requirements (FRs), design parameters (DPs), and process variables, respectively. Corresponding to these distinctive domains, the development of a PFA involves the following consecutive stages. 
BPMJ

4,1

16

Figure 3.

Structural implications of a PFA

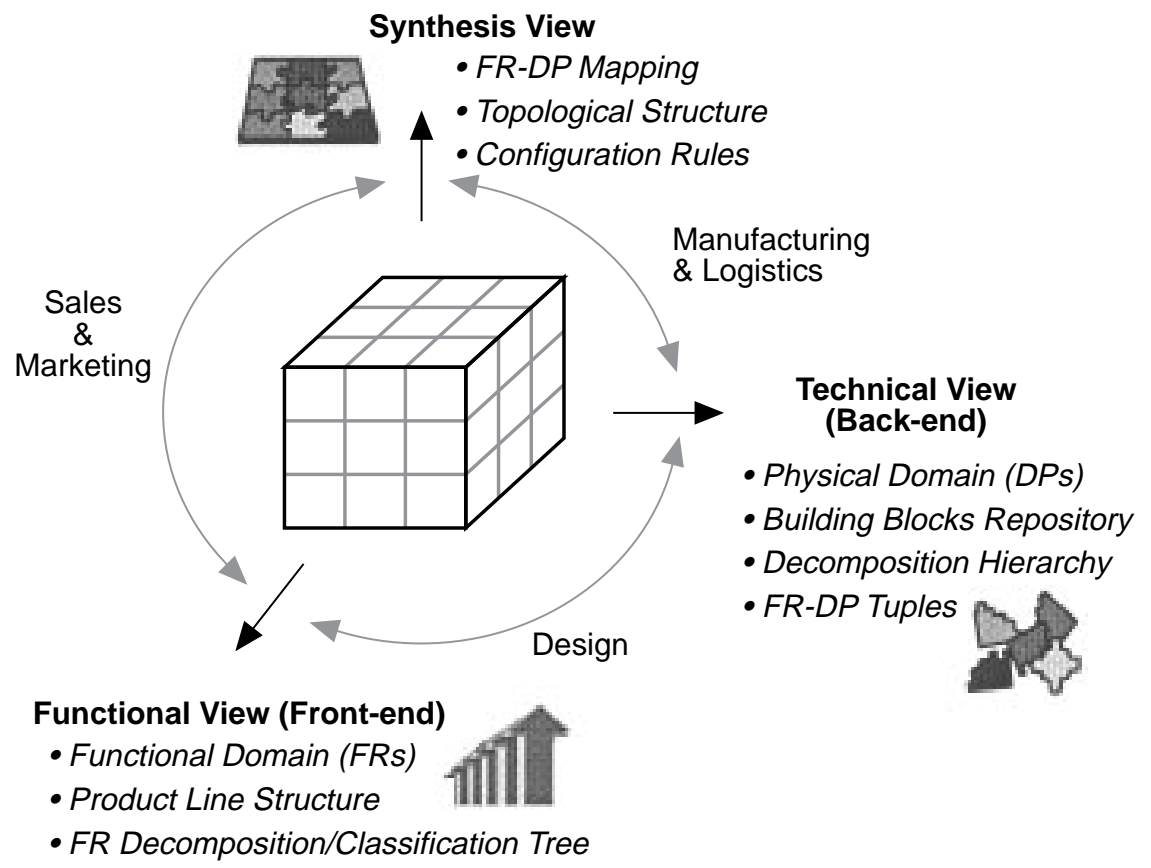

5.1 Customer-focused product requirement analysis

In the functional view of a PFA, a rigorous product line structure depends on the gestalt analysis of product requirements, which starts from the investigation of customer profiles followed by explicating the underpinning patterns of customer needs. The following steps are suggested for systematic analysis of the product portfolio.

- FRs formulation. The FRs formulation lies in the customer and functional domains and starts from the definition of a set of aggregate $\mathrm{FR}$ features or variables with respect to the existing product portfolio. Semantics methods such as the KJ method (A ffinity diagram) and M PM (multipickup method) are the basis for discovering the underlying facts from affective language (Shiba et al., 1993). These methods have been developed and used in industry for identifying the voices of customers for individual products. A similar process is recommended to identify key factors in FRs and key constraints in design (Tseng and Jiao, 1997).

TheFRs formulation aims at developing a FR hierarchy generic to the existing product portfolio. The inter-relationships among $F R$ variables are refer red to as a FR hierarchy. Since FRs heavily depend on expert insight into products, the formulation of $F R$ inter-relationships can apply knowledge acquisition processes often used in the development of artificial intelligence systems to capture technology improvement, product migration, competition, new process introduction, production 
capacity change, and so on. In addition, design experts are interviewed to elicit expert heuristics on the technical aspects of product requirements. This is particularly important for industrial products.

- FRs instantiation. Once a FR hierarchy is established, the functional specifications of existing products can be mapped onto it and thus generate various FR instances to represent particular products. Due to the variation of customer needs, zero can be an acceptable value for specific FR variables.

- Data mining for eminent customer groups. Customer profiles are usually projected by a population of $F R$ instances. A pareto analysis helps to extract eminent customer needs by grouping FR instances into different classes according to the similarity of the embodied customer needs. The consideration in pareto analysis involves the quantity measurement characterized by the demands and/or production volume. The understanding of customer profiles and focused segmentation pose conducive guidelines and constraints on product line structuring in PFA development. Because some FRs cannot be represented in numerical form, statistical correlation will not be possible. Hence, tools that are capable of performing symbolic processing will be needed to identify the proximity of FRs so that the functional specifications for a product family can be inferred.

- Functional classification. The underpinning patterns of customer needs are embodied by the classification of various product specifications, namely functional classification. In functional classification, FRs are adopted as classification parameters to describe both the characteristics of an entire product family and those of individual variants within the family. The key to functional classification is how to identify boundaries between classes of customer needs in terms of the range of the value distributions of $F R$ variables. For this purpose, clustering techniques are applicable (A nderberg, 1973).

In our research, conceptual clustering (Stepp, 1984) has been applied. It classifies functional specifications (i.e. FR instances) based on concept membership, that is, based on whether a particular FR instance and all others in a functional class (a functional variant) can be described by a single concept (characterized by the $F R$ variable of that class). In the CLUSTER/2 program (Stepp, 1984), leaves of a FR hierarchy are employed as the classifying attributes (variables) to describe various functional classes (variants). The volumes of $F R$ instances derived from respective customer demands are weighted in determining concept memberships so as to incorporate volume information in functional classification (T seng and Jiao, 1997).

- Product strategy exploration. In addition to a thorough understanding of current product spectrum, product migration should be considered to 
BPM J

4,1

18

proactively anticipate future product line structure. This involves assessment of product strategy according to technological evolution, competition, market trends, etc. Obviously, this task is domaindependent and qualitative in nature. A fter all, adding product strategy to product line structuring is important for a robust PFA (Roberts and Meyer, 1991).

5.2 Reusability-emphasized building blocks identification

A s the basic elements of a PFA, building blocks are referred to as various sets of DPs with their specific values corresponding to and satisfying different functional specifications. The identification of building blocks depends on the clustering of designs that involves the selection of DPs, their tentative FRs, the understanding of FR-DP mapping, and the boundaries between various design instances. Systematic steps are suggested as following to identify building blocks in design.

Design representation. A ccording to axiomatic design theory (Suh, 1990), decision structure inherent in the design process involves the definition of DPs as an explicit means to satisfy a progressively decomposed set of requirements and to provide detailed, concept-level associations of requirements to available options. FRs are said to exist in the abstract functional domain and generally have a multitude of possible solutions that exist in the physical domain. DPs are selected based on their ability to fulfill FRs. Processes such as the zig-zagging decomposition process proposed by Suh (1990) are very useful in identifying these parameters in individual products. A DP hierarchy is formulated to describe DPs and their inter-relationships. The mapping relationships in design are depicted by a design matrix which links a FR hierarchy and a DP hierarchy.

Design instantiation. Once the FR and DP hierarchies and the design matrix are established, all the existing product designs can be mapped into design instances. Each design is represented by the instance of a FR-DP tuple that consists of a FR vector and the corresponding DP vector.

Clustering DP variation for building blocks. Conventionally, DPs are defined for each individual product to fulfill a set of FRs. For the design of a product family, in essence, a few segments of DPs should be adopted to cover the same $F R s$ that would have required a continuum of DPs. By limiting the spread of DP variation, a few selected sets of DPs would be able to fulfill the same spectrum of customer needs.

Due to the complexity of design mapping, the clustering of design variants cannot normally be done by intuition. A number of tools used for clustering analysis have been reported (A nderberg, 1973). However, originating from the nature of design, many FRs and DPs involve a combination of numerical values and symbolic descriptions, where inductive learning techniques are applicable to sort out the similarities and to identify groupings (Lu and Tcheng, 1990). In our research, fuzzy clustering analysis is found suitable to identify building blocks in design. Detailed descriptions on fuzzy clustering analysis are presented in (Zimmermann, 1991). 
Granularity trade-off of building blocks. Economically, the cost of additional building blocks and the obtained customization need to reach a balance at the right level of the granularity of the product family. The question here is the economical justification of building blocks' ramifications. A $n$ approximate tool, advocated by Ishii et al. (1995), can be employed to support decision making in the economical evaluation of building blocks. It basically quantifies the importance and cost of each building block and uses an importance-cost plot to position different building blocks for conducting granularity trade-off.

\subsection{Product family architecture synthesis}

The PFA synthesis mechanism concerns three types of design knowledge, namely FR-BB correspondence, topological structure, and configuration rules. The following steps are suggested to extract synthesis knowledge for a PFA .

Benchmarking products with respect to building blocks. Once a set of building blocks is obtained, all existing products are instantiated and analyzed in terms of different kinds of building blocks so as to obtain consistent mapping relationships between functional specifications and building blocks (FR-BB correspondence), as well as the configuration knowledge for individual products.

Formulating topological structure. The next step is to establish the interrelationships among building blocks regarding product configuration. A straightforward method is to apply the DP tree structure derived from the DP decomposition. In addition, the overall design concepts need to be explicated and formulated according to domain knowledge.

Inducing configuration rules. With various building blocks and different product families, the sequences of design mapping from customer needs to design solutions can be many. In order to identify the preferred sequences, the sequential learning technique implicated by axiomatic design theory (Suh, 1990) provides a structural mapping for cascading variables along the customer domain, functional domain and physical domain. In addition, domain specific constraints are explored regarding the coupling parameters, which need more coordination efforts in the design process.

\section{A case study}

The power supply is a key component in electronic products, such as telephone switching PBX, stereo equipment, computers, and instrumentation. A power supply company under our investigation offers the standard product covering more than 1,200 varieties, along with a wide range of customized products. Because of the growing varieties, the company is constantly challenged to achieve responsiveness, flexibility, and cost. There is a significant amount of engineering expense involved in meeting individual customers' needs. In this business sector, lead time is a major competitive advantage, and customers are often willing to explore possible alternatives. Facing the seemingly conflicting goals, i.e. satisfying individual customer requirements vs. being a low cost producer, the company has recognized mass customization as a potential 
solution to its business dilemma. The CDF M C methodology has been applied to 4,1 this study of mass customization realization.

First, domain design knowledge is acquired through comprehensive interviews with experts and is formulated according to axiomatic design theory. Tables I and II givethesimplified FR and DP hierarchies for power supply design. $M$ ore than 300 existing products are mapped into their FR instances and DP instances. These historical design data provide the basis for PFA development.

The underpinning patterns of customer needs are extracted through functional classification. Table III gives an example of employing $F$ Rs as parameters in functional classification, where different values of classification parameters, i.e. their instances, manifest the differentiation of functional variants. Based on the results of functional classification, the product line is constructed by further incorporating considerations of technological trends, competition, and process capabilities. A combined decomposition/classification tree is employed to represent the product line structure for power supplies, as shown in Figure 4. As a type of industrial product, a power supply features a

Table I.

FR hierarchy for power supplies

\begin{tabular}{llll}
\hline Descriptivelevel & Generic level & Terminology level & Engineering level \\
\hline FR1: & FR11: & FR111: Line voltage & FR1111: Voltage range \\
$\begin{array}{l}\text { Used } \\
\text { in }\end{array}$ & Operating range & FR112: Input surge current & \\
$\begin{array}{lll}\text { what } \\
\text { country }\end{array}$ & FR12: Protection & FR113: Line transient & \\
(Input requirement) & & FR122: Power-line & FR1221: Brown-out \\
& & disturbance & FR1222: Drop-out \\
& & FR123: RFI/surge & \\
& & suppression &
\end{tabular}

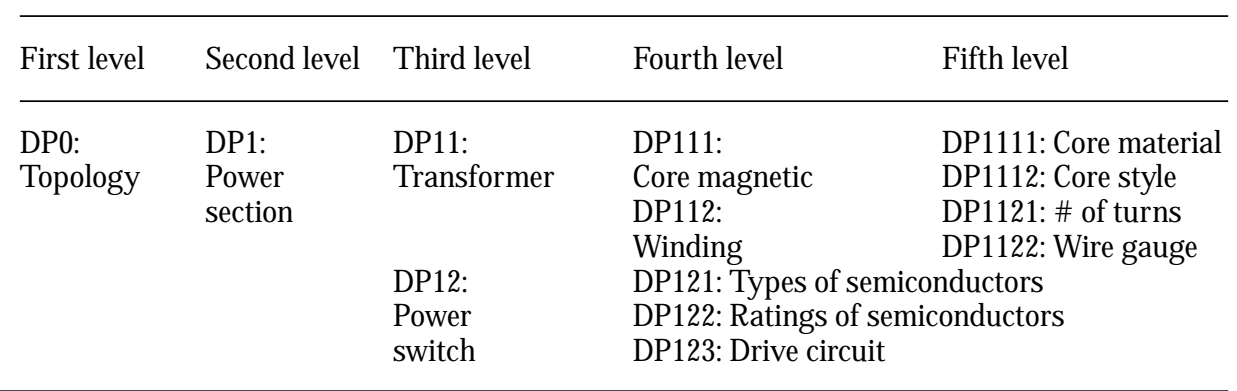

\section{Table II.}

DP hierarchy for power supplies

\section{Classification parameters $=\mathrm{FRS}$}

Total power
Number of outputs
PFC
Specifications for families

Specifications for families
Classification parameter values $=\mathrm{FRs}$ instances

Table III.

Classification of the functional specifications of power supplies

\begin{tabular}{cccc}
$<200 \mathrm{~W}$ & $>200 \mathrm{~W}$ & $\&<1000 \mathrm{~W}$ & $>1000 \mathrm{~W}$ \\
Single & Dual & Triple & $>3$ \\
Yes & No & \\
\multicolumn{3}{c}{ Individual specifications for variants }
\end{tabular}




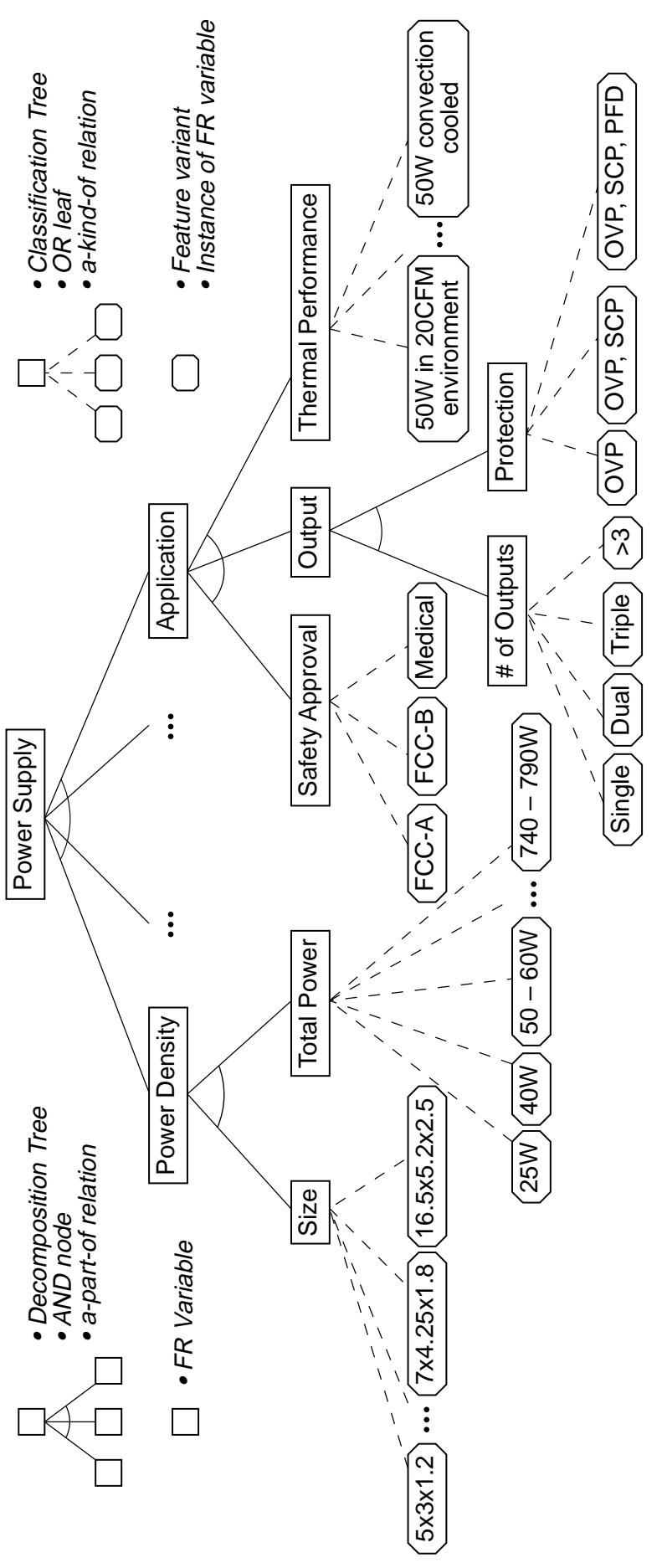


BPMJ number of complex customer requirements, resulting in diverse variations. A 4,1 FRs-based functional classification provides a structural method to characterize the differentiation of power supply products, which enhances the clear understanding of customer requirements. From a functional viewpoint, the customer requirements or product features of a power supply can be described by a FR vector, i.e. $\overline{F R}$ power supply $=\{$ size, total power, safety approval, \# of outputs, protection, thermal performance\}. The functional specification of a particular power supply is represented as an instance of this vector, e.g. $\overline{\mathrm{FR}}_{\mathrm{nfs} 40-7608}=\{5 \times 3 \times 1.2$ in size, $40 \mathrm{~W}$ output power, $\mathrm{FCC}$ class B safety requirement, triple outputs, over voltage protection and short circuit protection, cooling by 50W in 20CF M environment $\}$. In such a way, the functional classification performs as a structural formalism to characterize product line differentiation for power supply products.

Building blocks are identified by clustering DP variations with respect to specific FR-DP mapping relationships. The decision implications for identifying appropriate building blocks are provided based on experts' knowledge as a result of understanding the characteristics of the clustered classes. Table IV gives an example of building blocks and the FR-DP mapping relationship.

Various circuitry topologies highlight the overall design concept to guide synthesis of power supply designs based on the building blocks. A topology determines the way in which the power holding parts of a power supply are configured.

In this case study, the impact of CDFMC on the company and its managerial implications are multifold. CDFMC embodies the collaboration efforts between sales and marketing and engineering to define new product families with respect to product portfolio planning. Through market research, marketing clusters customer requirements into patterns reflected by a product line structure that facilitates the engineering department to position various products and to incorporate competition concerns (from marketing), new product technology

Table IV.

A n example of building blocks in power supply design

\begin{tabular}{|c|c|c|c|}
\hline \multirow{3}{*}{$\begin{array}{l}\text { Building block } \\
\text { variants } \\
\text { BBk }\end{array}$} & \multicolumn{3}{|c|}{$\begin{array}{l}\text { FR-DP mapping relationship: } F R k=f_{F R K}(D P k 1, D P k 2) \\
\text { Building blocks representation: } B B k=(F R k ; D P k 1, D P k 2)\end{array}$} \\
\hline & $\mathrm{FRk}=$ & DPk = "magne & core of transformer \\
\hline & output power (W) & DPk1 = core type & DPk2 = core size $(\mathrm{mm})$ \\
\hline MPP-A & $<5$ & MPP Toroid & 16 (diameter) \\
\hline MPP-B & $<25$ & MPP Toroid & 20 (diameter) \\
\hline MPP-C & $<50$ & MPP Toroid & 30 (diameter) \\
\hline MPP-D & $<100$ & MPP Toroid & 38 (diameter) \\
\hline MPP-E & $<250$ & MPP Toroid & 51 (diameter) \\
\hline EEL-A & $<5$ & $E-E, E-L$, etc. Core & 11 (each side) \\
\hline EEL-B & $<25$ & $E-E, E-L$, etc. Core & 30 (each side \\
\hline EEL-C & $<50$ & E-E, E-L, etc. Core & 30 (each side) \\
\hline EEL-D & $<100$ & $E-E, E-L$, etc. Core & 47 (each side) \\
\hline EEL-E & $<250$ & $E-E, E-L$, etc. Core & 60 (each side) \\
\hline
\end{tabular}


(from engineering), new process technology (from manufacturing), and new packaging (industrial design), etc. The sales department performs some metadesign, such as configuration, to conform to customer's expectations in addition to exploring market niches. Design function shifts from individual product design to product family design in response to technology migration, manufacturing process evolution, or customer requirement turbulence. $M$ anufacturing focuses on the reuse of tooling, set-up, process knowledge, and so on, along with building blocks identification and interface assessment.

Concurrent design for mass customization

\section{Conclusions}

This paper proposes an approach to CDFMC. In order to facilitate the maximization of customer value via matching the scopeneeds of customers and the capabilities of the firm to increase scale, systematic steps are developed to formulate a PFA. Based on machine learning techniques, the clusters of DPs are identified as building blocks to fulfill the FRs and optimize the common denominators in design solutions.

CDFMC integrates entrepreneurial development with a disciplined systematic approach, thus forming a context-coherent integration platform for simultaneous product development with these benefits:

- a platform developed with a systematic approach is more stable and reduces reactive changes;

- products can be developed very quickly by making variations from a PFA. Customer needs can be quickly addressed with very little slack time for either customers or product developers to make changes;

- the migration path of the platform will evolve through incorporating customer needs, process experience, and learning, hence making possible the sharing of knowledge across different products; and

- PFA allows a product specification from both functional and technical views, which enhances sales and manufacturing coordination.

\section{References}

A nderberg, M.R. (1973), Clustering A nalysis for A pplications, A cademic Press, N ew York, NY.

Ishii, K., Juengel, C. and E ubanks, C.F. (1995), "Design for product variety: key to product line structuring", 9th International Conference on Design Theory and M ethodology, DE, Vol. 83 No. 2 pp. 499-506, A SME, New York, NY.

Kotha, S. (1994), "M ass customization: the new frontier in business competition", A cademy of M anagement Review, Vol. 19 No. 3, pp. 588-92.

Kotha, S. (1995), "Mass customization: implementing the emerging paradigm for competitive advantages", Strategic M anagement Journal, Vol. 16 (special issue), Summer, pp. 21-42.

Lau, R.S. (1995), "M ass customization: the next industrial revolution", Industrial Management, Vol. 37 No. 5, pp. 18-19.

Lu, S.C-Y. and Tcheng, D. (1990), "Building layered models to support engineering decision making: A machine learning approach", Journal of Engineering for Industry, Vol. 113 No. 1, pp. 1-9. 
BPMJ

4,1

Meyer, M.H. and Utterback, J.M. (1993), "T he product family and the dynamics of core capability", Sloan M anagement Review, Vol. 34 No. 3, pp. 29-47.

Molina, A ., A i-A shaab, A.H., Ellis, T.I.A . and Young, R.I.M . (1995), "A review of computer-aided simultaneous engineering systems", Research in Engineering Design, Vol. 7 No. 1, pp. 38-63.

Pine II, B.J. (1993), M ass Customization: The New Frontier in Business Competition, Harvard Business School Press, Boston, MA .

Roberts, E.B. and M eyer, M.H. (1991), "Product strategy and corporate success", IEEE Engineering M anagement Review, Vol. 19 No. 1, pp. 4-18.

Shiba, S., Graham, A . and Walden, D. (1993), A N ew A merican T QM, Productivity Press, Portland, OR.

Sohlenius, G. (1992), "Concurrent engineering”, A nnals of the CIRP, Vol. 41 No. 2, pp. 645-56.

Stepp, R. (1984), "Conjunctive conceptual clustering: a methodology and experiments", PhD thesis, Report No. UIUCDCS-R-84-1189, Department of Computer Science, University of Illinois at Urban-Champaign.

Subrahmanian, E., Westerberg, A . and Podnar, G. (1991), "Towards a shared computational environment for engineering design", in Sriram, D., Logcher, R. and Fukuda, S. (E ds), Computer Aided Cooperative Product Development, Springer-Verlag, Berlin.

Suh, N.P. (1990), T he Principles of Design, Oxford University Press, New York, NY.

T seng, M.M. and Jiao, J. (1996), "Design for mass customization", A nnals of the CIRP, Vol. 45 No. 1, pp. 153-6.

Tseng, M.M. and Jiao, J. (1997), "A variant approach to product definition by recognizing functional requirement patterns", Journal of Engineering Design, Vol. 8 No. 4, pp. 329-40.

Zimmermann, H.J. (1991), Fuzzy Set Theory and its A pplications, Kluwer A cademic Publishers, Boston, MA . 